\title{
Téoros
}

Revue de recherche en tourisme

\section{Pour un corridor touristico-industriel en Estrie}

\section{Roger Nadeau et Jean-Louis Blanchette}

Volume 5, numéro 1, mars 1986

Tourisme et régions

URI : https://id.erudit.org/iderudit/1080587ar

DOI : https://doi.org/10.7202/1080587ar

Aller au sommaire du numéro

Éditeur(s)

Université du Québec à Montréal

ISSN

0712-8657 (imprimé)

1923-2705 (numérique)

Découvrir la revue

Citer cet article

Nadeau, R. \& Blanchette, J.-L. (1986). Pour un corridor touristico-industriel en Estrie. Téoros, 5(1), 13-15. https://doi.org/10.7202/1080587ar d'utilisation que vous pouvez consulter en ligne.

https://apropos.erudit.org/fr/usagers/politique-dutilisation/ 


\title{
Pour un corridor touristico-industriel en Estrie
}

\author{
par Roger Nadeau et Jean-Louis Blanchette*
}

\section{La naissance d'une idée}

En 1972, l'Office de Planification et de Développement du Québec lançait une vase mission de planification en Estrie et 18 rapports de recherche plus tard. l'Office pouvait diagnostiquer que la région était sérieusement malade et qu'il fallait intervenir rapidement pour éviter que l'état de santé général de la patiente ne se détériore davantage. Les deux remèdes de croissance prescrits par les spécialistes furent les suivants: procéder sans délais à une reconversion industrielle et développer l'énorme potentiel touristique de la région.

Qu'en est-il aujourd 'hui, 12 ou 13 ans plus tard? Disons tout de suite qu'il apparait clairement que le processus de developpement de 1 "industrie touristique est enclanché solidement, surtout grảce à l'élan, à l'envol qu'il s'est donné au début des années ' 80 . Lors du Sommet socioéconomique de l'Estrie, tenu en janvier 1985,37 des 45 millions $\$$ d'engagements gouvernementaux l'ont été dans le secteur du tourisme, ce qui témoigne du dynamisme de ce champ d'activité qui devrait créer plus de 7000 emplois au cours des cinq prochaines années, selon les évaluations ministérielles. Oui, le tourisme est en train de prendre sa place en Estrie; qu'on en juge par les quelques projets qui suivent: 2205 millions pour la Station touristique Intematinale Magog-Orford, $80 \$$ millions a la station touristique de Bromont, 40 \$ millions à Orford, 405 millions aे Owl's Head, $20 \$$ millions au Domaine StLaurent, etc...etc. C'est ce qui permettait au ministère du Tourisme d'écrire en octobre 1984 que l'Estrie connaissait sle plus haut coefficient de projets et d'investissements majeurs dans le secteur du tourisme au Quebec* ${ }^{a}$ Et cette activité donne des résultats concluants sur le terrain: l'Estric accueillait 1.3 millions de voyages/personnes en 1984, ce qui représentait $14.3 \%$ du total québecois (comparé à $18.3 \%$ pour les Laurentides). ${ }^{(2)}$ Dans l'ensemble donc, même s'il reste encore beaucoup à faire,

Roger Nadeau est professeur au departemen de gtographie de rUnivarsite de Sherbrooke Jear-Louis Blanchette a terminé une maitrise en gtographe a certe institution, avec une these sur le concept dissute ici. on peut dire que l'Estrie est en train de prendre sa place sur l'échiquier du tourisme québécois.

De son côté, le projet de reconversion industrielle tire sérieusement de la patte. Dans l'esprit des planificateurs, cette reconversion devait consister à combler les effets du déclin de l'industrie textile (de loin le plus important secteur manufacturier de l'Estrie) par la transformation de l'amiante en sol québecois... et estrien. Or, l'amiante a très mauvaise mine depuis bientôt une décennie, au point que son contingent de travailleurs est passé de près de 9000 a moins de 2000 présentement. Du cóté du textile, le secteur continue de se débobiner et l'annonce de l'abandon d'une partie importante du contingentement à l'importation ainsi que la perspective d'un libreechange Canada - Etats-Unis sont susceptibles d'accélèrer les difficultés que connait cette industrie.

C'est dans ce contexte que l'on s'est rappelé qu'IBM avait expliqué son implantation à Bromont par la qualité du milieu de vie offerte par le cadre physique de la région. On vient de donner la méme explication pour Hyundai ou pour TIE Communications à Sherbrooke. Ces exemples confirment ce que nous apprennent les traités pornant sur les facteurs de localisation industrielle qui signalent que la qualité de wie offerte par les régions a haut potentiel touristique constitue un attrait important, surtout pour les entreprises de pointe ou de haute technologie. D'autre part, les experiences d'aménagement régional d'envergure, tels celles du Tennessee Valley Autority ou du Languedoc-Roussillon ont clairement démontré que le tourisme seul peut rarement faire vivre une région et que l'industrie touristique se portera bien dans la mesure oú les autres secteurs de l'économie feront aussi preuve de dynamisme. Et l'avenir des régions et des pays avancés se situe dans la haute technologie, selon la plupart des auteurs, ${ }^{\text {(3) }}$ ce qui a incité I'ATE à produire un premier et bref document de refflexion pour associer toutes ces composantes régionales, donnant ainsi naissance à l'idée d'un faxe touristicoindustriel en Estrie (4) Suite à des reflexions et à des discussions sur le sujet. M. Jean-Louis Blanchette décidait d'en faire son sujet de thèse de mailtrise, sous la direction de Roger Nadeau et de Rejean Beaudoin. Ce texte a pour but de faire part des réflexions et démarches en cours.

\section{Une problématique estrienne}

Comme l'affirme le rapport GaucherPringle, l'Estrie possède, grảce à la région Magog-Orford, I'un des rares sites du Québec ou sont présentés les trois facteurs essentiels au developpement d'une station touristique internationale exploitable en toute saison, soit un plan d'eau pour les sports nautiques, des montagnes pour le ski et une zone urbaine pour les activités commerciales et culturelles. ${ }^{(5)}$ Ainsi si l'on peut privilégier lè développement du tourisme estrien non seulement autour de ce póle, mais aussi près de Sutton, Bromont et de la région en général, celle-ci sera en meilleure position pour faire face au marché que constituent les 23 millions d'habi tants résidant dans un rayon de $500 \mathrm{~km}$. Ces atouts à exploiter sont d'autant plus importants si l'on tient compte du fait qu'une bonne partie de la population active profite de ses vacances par petites périodes de 3 ou 4 jours, réparties sur toute l'année. D'oủ I'importance qu'il y a à trouver sur place des possibilités de sports d hiver ou autres activités susceptibles de plaire aux visiteurs. Il ne fait aucun doute que l'Estric profite grandement des retombées économiques que génère l'ensemble des activités touristiques, quoiqu'il soit assez difficile d'en évaluer $1^{\dagger}$ impact précis.

Dans un autre ordre d'idées, on constate que les organisations régionales de promotion industrielle telles que les chambres de commerce, commissariats industriels, mairies... cherchent de plus en plus à attirer dans la région estrienne un type d'industrie qui semble convoité par nombre de régions tant au Québec qu'ailleurs au Canada: il s'agit de l'industrie de pointe. Cet engouement pour la haute technologie. prise au sens large du terme, s'explique en grande partie par le fait que les industries de ce secteur effectuent, en génerral, passablement de recherche et de développement, sont peu ou non-polluantes et 
constituent d'excellents moyens de croissance et de promotion industrielles régionales.

Les industries de pointe se développent plus rapidement que les autres et constituent. dans la plupart des cas, «des illots de pros. périté dans un paysage assez gris de diffi. cultés économiques. Ces industries ont certes leur part de problemes, liés à la conjoncture économique, mais il n'en demeure pas moins qu'elles génèrent des retombées économiques, d'autant plus importantes lorsque ces industries proviennent d'initiatives locales. De plus, la majorité des salaires payés sont supérieurs à la moyenne, d'où des renurées fiscales meilleures pour les régions qui les accueillent et une activité économique induite dans les villes qui les logent.

Le cas de Bromont, d'une part, illustre bien ce phénomène puisque quelques-uns des plus importants employeurs régionaux, tels que Général Électrique, IBM et Mitel, tous trois oeuvrant dans des secteurs de pointe, sont à Bromont. Ces industries sont situées dans un parc industriel spécifique à la haute technologie. Au Québec, seuls Bromont, Hull, Montréal et Sherbrooke possèdent un tel parc. IBM, avec ses 1500 employés a comme politique d'encourager l'achat dans la région ce qui multiplie les retombées économiques: services professionnels, papeterie, meubles de bureaux, accessoires electriques, voitures et camions, etc. De plus l'arrivée du constructeur automobile sud coréen Hyundai, dont l'usine devrait compter tout près de 1200 employés, aura des répercussions positives, tout particulièrement au niveau de la sous-traitance.

D'autre part Sherbrooke tend également à développer son économie vers de nouveaux domaines, dont celui des secteurs de pointe. Ces derniers contrebalancent quelque peu les pertes d'emplois, notamment dans l'industrie du textile qui occupe toujours une place prépondérante au sein de la région. D'ailleurs pour faire face aux problèmes dus à la concurrence internationale. les industries du textile, entre autres Dominion Textile, sont parmi celles qui ont investi le plus au cours des dernières années dans le but d'acquérir les plus récentes techniques de pointe. Il est donc nécessaire de ne point sacrifier ce secteur de l'économie régionale en dépit des problèmes auxquels il doit faire face. Etant l'une des sept régions désignés par l'OCRI (Office canadien pour un renouveau industriel), la région de Magog-Orford-Sherbrooke a plus particulièrement brillé par son dynamisme en récoltant près de $35 \%$ des subventions accordées aux zones. C'est gráce, en partie, à ce programme mis en place il $\mathrm{y}$ a quatre ans, que le visage économique de Sherbrooke a subi une certaine permutation, la diversification de la structure industrielle étant bien amorcée. De plus, la présence à l'Université de Sherbrooke de la Société de micro-électronique de Sherbrooke (SMIS) qui emploie 18 ingénieurs et techniciens oeuvrant dans le domaine de la micro-électronique, est un atout nonnégligeable. A preuve, la société C-MAC Inc., une nouvelle entreprise formée par une firme de Montréal de mème qu'une douzaine d'investisseurs de Sherbrooke, a annoncé récemment la construction d"une usine pour la fabrication de circuits hybrides à Sherbrooke. Le choix de ce site est dû en grande partie à la présence du SMIS. ${ }^{\left|{ }^{6}\right|}$

\section{Des développements convergents}

Ainsi l'avenir économique de l'Estrie semble s'orienter, du moins en partie. vers le développement des activités de loisirs et du tourisme d'une part, de méme que vers l'expansion de l'industrie à haut potentie technologique d'autre part. On constate toutefois que ces deux secteurs d'activités. soit le tourisme, ce vaste mouvement qui englobe l'ensemble de l'activité socioéconomique des personnes aptes à quitter temporairement, pour leur agrément, leur propre milieu habituel, ainsi que le secteur des industries de pointe, sont à maintes reprises perçues comme étant incompatibles entre eux, c'est-à-dire qu'ils ne peuvent être associés ensemble et qu'ils doivent plutớt évoluer et se développer d'une manière autonome. Cette perception se vérifie par le fait que peu d'études exhaustives ont jusqu'à présent été entreprises à ce sujet. Qu'en est-il exactement? La qualité de vie d'une région alliée à un développement touristique adéquat peuvent-ils être considérés comme facteurs importants de promotion et de développement industriels? Si oui, à quel niveau? Quelle est l'importance du facteur qualité de vie par rapport à l'ensemble des autres facteurs de localisation dont tiennent compte des industries de pointe?

En tenant compte des observations précédentes, M. Réjean Beaudoin a mis l'accent sur un nouveau concept qui ferait de la région de Sherbrooke-Magog-OrfordBromont un axe touristico-industriel unique au Québec. Nous croyons que la région comprise à l'intérieur de cet axe posséde tout le potentiel de base requis pour devenir un modèle concret d'application pour le Québec, dominé par une qualité de vie favorisant le culturo-récréo-tourisme et la haute technologie. Ainsi, notre troisième objectif sera de créer l'axe mentionné cihaut et d'évaluer la capacité de ce dernier de rencontrer les exigences et les besoins des industries de pointe. La zone prioritaire de notre axe sera limitée a la région sise entre les villes de Bromont et Sherbrooke, sans toutefois exclure des endroits en périphérie tels que Lennoxville, Massawippi. la région de Owl's Head ainsi que quelques autres, qui par leurs caractéristiques géographiques font partie intégrante du milieu. II serait donc tout indiqué de tenir comple de ces endroits lorsque viendra le moment d'évaluer le potentiel de notre axe.

L'un des póles, Sherbrooke, est une agglomération qui, d'une part, possède une structure industrielle relativement bien diversifiée tendant vers les secteurs de pointe, et d'autre part, une qualité de vie remarquable. De plus la présence de deux institutions universitaires, l'une francophone, l'autre anglophone, à proximité de Sherbrooke constitue un atout non négligeable. L'autre pôle, Bromont, semble appeler à devenir un centre regroupant diverses industries de pointe dans un environnement favorisant le développement des activités de loisir. Au centre de l'axe se trouvent Magog-Orford de méme que la région environnante, toutes deux étant appelées à former une zone orientée vers l'activité du tourisme et de la villégiature. Comme l'affirme Alain Duhamel - le tourisme et la villegiature ont pris un esor considerable. accaparant une part substantielle du développement économique de la MRC de Memphrémagog. L'une des principales orientations que crée la proposition d ame. nagement de cette $M R C$ inscrit le dévelop. pement du tourisme, non seulement dans la station touristique Magog-Orford, mats aussi dans d'autres secteurs de son territoire où le caractère champêtre des liewx s'affirmes, ${ }^{77}$ On y prévoit des investissements très importants au cours des prochaines années. La présence de ces atouts. croyons nous, explique en grande partie le potentiel global de qualité susceptible d'étaler le climat de vie conforme aux besoin.s des visiteurs et des industriels des secteurs de pointe. Le rapport Gaucher-Pringle affirme que sla mise en valeur du potentiel touristique et le's retombées économiques qui en découleni peuvent jouer un róle imporiant dans la reconversion industrielle de la zones, 18

À la lumière des quelques lectures effectuées nous sommes en mesure d'élaborer et de vérifier, d'une part. l'équation ou I'hypothèse selon laquelle une région offrant une qualité de vie supérieure par des installations touristiques adéquates et un environnement stimulant est un facteur important qui sera pris en considération par une industrie de pointe, lorsque viendra le moment de s'implanter dans une région.

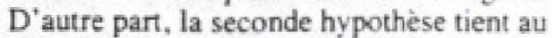
fait que le développement touristique d'une région donnée peut être un facteur de promotion et de relance industrielle non négligeable.

La confirmation ou l'infirmation de nos hypothèses se vérifiera par les résultats d'une enquête auprès de diverses industries oeuvrant chacune dans leur domaine de pointe respectif. Les résultats nous serviront par la suite à analyser et évaluer la capacité qu'a notre axe de rencontrer les exigences des entreprises de pointe face à leur environnement. 


\section{Un approche neuve et peu connue}

Comme il en a été fait mention précédemment. peu d'études ont jusqu 'à présent tenté de répondre à ces questions d'une manière précise. Comme l'affirme Alister Mathieson, wla lintérature fair très peu référence aux changements de la structure indus= trielle d'une région qui pourraient être dus au développement de l'industrie touristque ${ }^{\text {(9) }}$ Par contre, certains articles relativement récents ont abordé le sujet de façon indirecte. Ainsi on observe aujourd "hui que les industries à la recherche d'un site ne tiennent plus compte exclu= sivement, comme par le passé. des facteurs quantitatifs (avantages fiscaux, voies d'accès, proximité du marché et des res= sources naturelles...). Certes, ces derniers sont toujours pris en considération, mais il appert que les facteurs qualitatifs exercent une influence de plus en plus prépondérante lorsque vient le moment d'effectuer une implantation industrielle. ${ }^{10}$ En dépit du fait que ces derniers soient difficilement quantifiables, ils revêtent une importance certaine pour nombre d'industries. Ces facteurs qualitatifs sont de plusieurs ordres: qualité du climat et de la main-d'oeuvre, présence d'institutions universitaires répondant aux besoins des industries, attributs culturels et esthétiques d'une région, choix d'activités sportives, dynamisme local... On observe d'ailleurs depuis quelques années une nouvelle tendance voulant que les facteurs qualitatifs soient de plus en plus pris en considération, le contenu de divers articles traitant de la question confirmant, en général, cette présomption.

Ainsi, l'érnergence d'une nouvelle structure industrielle axée sur le développement de l'industrie de pointe a fait en sorte que de nouveaux facteurs (qualitatifs), négligeables il y a quelques années, ne le sont plus aujourd'hui et ce, au détriment de facteurs traditionnels (quantitatifs). Un autre auteur. Mark Larsen, parlant du mème phénomène ajoute: these individuals recognized that fondamental economic changes were taking place: science-based industry and a technically trained labor force are merging as key determinants in regional growth supplementing, if not replacing, the influence of such natural resources, as proximity to cheap water transponation, raw materials. central markets, and the availability of semi-skilled labor $\$$,

Il semble donc que l'importance du cadre de vie de même que la situation géographique soient des phénomènes assez récents et qui tendent à prendre de plus en plus d'ampleur. ${ }^{112 .}$ On a observé aux Etats-Unis de même qu'en Europe, la tendance qu'ont les industries de pointe à se regrouper non pas dans des grands centres urbains et industriels, mais plutôt dans des régions *neuves- offrant des avantages d'une infrastructure d'accueil aux plans physique

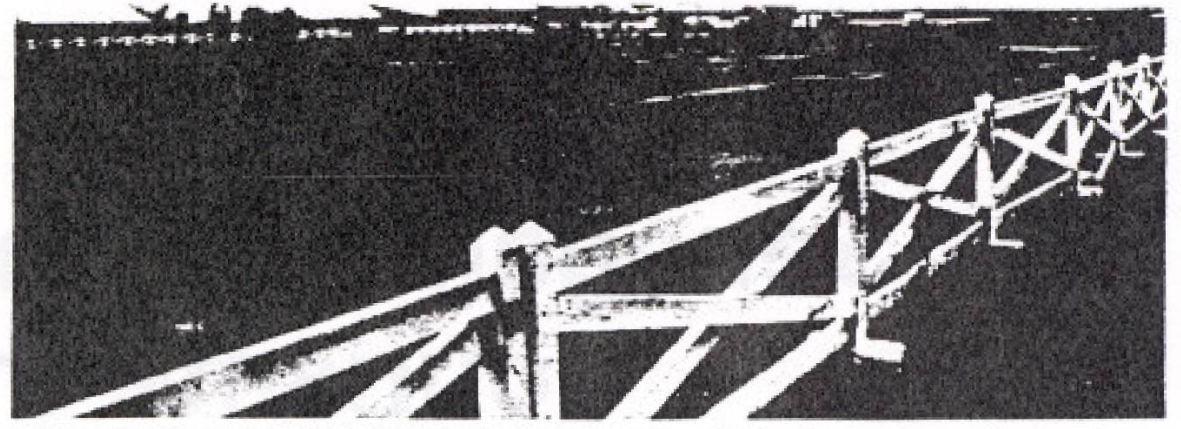

L'hypodrome de Bromont: un atout pour cette localité qui témoigne d'un style de vie "sgráabie"

(espaces disponibles à prix abordables,..) et scientifique (proximité d"institutions unjversitaires possédant des orientations scientifiques et technologiques identifiées, ou d'instituts ou laboratoire importants, gouvernementaux ou privés). Ces entreprises recherchent également une localisation qui réponde aux aspirations des employés (scientifiques et techniciens) qu'elles désirent recruter; ces aspirations s'expriment en termes de qualité de vie du milieu aux plans culturel, éducationnel, de loisir et d'environnement. Ainsi toutes les industries de pointe installées à Boulder. Colorado, ont fait mention du fait que le climat ainsi que la beauté du paysage environnant constituaient deux des plus importants facteurs qui auraient influencé le choix effectué en faveur de cet endroit. ${ }^{13)}$ De mẻme, la présence, à proximité du parc scientifique de Valonne, près de Nice en France, d'un milieu géographique favorisant les activites de plein air a certes exercé une influence positive sur la venue d'industries désirant quitter l'agglomération de Paris. De plus, comme l'affirme le journal Les Affaires dans son édition consacrée à la région de Granby-Bromont, "la beauté physique de la région $n$ 'est pas érangère non plus d l'arirance qu'elle comporte pour les entreprises qui cherchent un envi= ronnement agréable pour inciter ses cadres à y démenager ${ }^{(14)}$ On se souviendra que l'argument avait été retenu par IBM en 1972 pour y installer son usine, les cadres spécialisés préférant habiter dans des régions où le style de vie est agréable (sports, absence de pollution, vie de campagne, etc) tout en n"habitant pas trop loin d'une grande ville. Un dernier exemple, celui du Virginia Travel Council qui écrit: * Aujourd hui. les possibilités économiques et sociales qui oni résulte de notre succès dans le monde du tourisme ont beaucoup aidé l'allure et la désirabilité de la Virginie en tant que territoire artitre pour de nouveaux développements industriels. (...) La progression de norre erat, au rang de Mecque touristique, a édifié le climat de vie et les conditions de travail requises par l'industrie désirable."

\section{Conclusion}

Bien que les études approfondies sur les relations tourisme - qualité de vie - haute technologie semblent à peu près inexistantes, plusieurs témoignages et expériences démontrent qu'il existe effectivement une forte affinité entre ces trois composantes. au point que I'Estrie entend résolument orienter une partie importante de son devenir dans ce sens. Mème les hommes et les femmes politiques de la région sont déja fort sensibilises à cette approche prometteuse qu'il nous faut continuer à explorer et à favoriser.

Nous profitons de l'occasion pour inviter les lecteurs(trices) de Téoros intéressés(ées) à cette question ou détenteurs d'idées, de références ou d'informations sur le sujet, a communiquer avec nous, afin de nous permettre de recueillir tout ce qui peut être pertinent à cette réflexion et à son opérationnalisation

Merci a l'avance.

\section{Notes et références}

(1) LEGER, Marcel; lettre adressede a l'Association Touristique de l'Estrie, le 26 octobre 1994

(2) NADEAU, Hoger: Le tounisme en Estrie, chapitre d'un liwre en préparation sur l'Estrie, Sherbrooke, tie 1985, 32 pages.

(3) NAISBITT, John: Les dix commandements de T'avenir (Megatrends): Montreal-Paris, Sand Pnmeur, 1982, 348 papes.

(4) BEAUDOIN, Rejean: L'axe touristico-industriel Sherbrooke-Magog-Orford: Sherbrooke, 21 fevrier 1985, 6 pages.

(5) Gaucher-Pringle Consultants Ltee, Rappon Geucher-Pringle. Septembre 1982, p. 111

(6) MCDOUGALL. Stephen! The Gazette. 14 detcembre 1985

(7) DUHAMEL, Alain: Le Devoir, 4 panvier 1986

(6) Guucher-Pringle Consultants Li6e, op.cit. p. 111

(9) MATHIESON, Alister- Tourism: aconomic. physical and social impacts, New York, Long. man. 1982, p. 85

(10) SCHMENNER. Roger W. Look bevand the obvious in plant location, Harrard Business Reviow, Vol 57-1, 1979 Jan, Feb. p. 132

111. LARSEN, Mark. A mode for the esrabishment of a university related research pack within a framewark of selected management pxinci ples, University oif Washington, 1970, p. 77

(12) Camment attrier les industries de painte. (Mis. sion scienifique de l'ambassade de France aux Etats-Unis), Problemes bconomiques, no 1.779 . 1982 , ค. 29

(13) Siwcon Valley takes the mountain arr, The Eoonomist, August 7, 19e2, p. 72

(14) PICHE, Claude: Journal hes Affaires. Cahier spe. cial - La region de Granbv-Bromont, 30 novem. bre 1985, p. 2

115) Virginia Travel Council: Building a Balion Dollars Travel Industry isoutce non specitiee 